\title{
The phase diagram of ice: A quasi-harmonic study based on a flexible water model
}

\author{
R. Ramírez, ${ }^{\text {a) }}$ N. Neuerburg, and C. P. Herrero \\ Instituto de Ciencia de Materiales de Madrid (ICMM), Consejo Superior de Investigaciones \\ Científicas (CSIC), Campus de Cantoblanco, 28049 Madrid, Spain
}

(Received 19 June 2013; accepted 6 August 2013; published online 23 August 2013)

\begin{abstract}
The phase diagram of ice is studied by a quasi-harmonic approximation. The free energy of all experimentally known ice phases has been calculated with the flexible q-TIP4P/F model of water. The only exception is the high pressure ice $\mathrm{X}$, in which the presence of symmetric $\mathrm{O}-\mathrm{H}-\mathrm{O}$ bonds prevents its modeling with this empirical interatomic potential. The simplicity of our approach allows us to study ice phases at state points of the $T-P$ plane that have been omitted in previous simulations using free energy methods based on thermodynamic integration. The effect in the phase diagram of averaging the proton disorder that appears in several ice phases has been studied. It is found particularly relevant for ice III, at least for cell sizes typically used in phase coexistence simulations. New insight into the capability of the employed water model to describe the coexistence of ice phases is presented. We find that the $\mathrm{H}$-ordered ices IX and XIV, as well as the H-disordered ice XII, are particularly stable for this water model. This fact disagrees with experimental data. The unexpected large stability of ice IX is a property related to the TIP4P-character of the water model. Only after omission of these three stable ice phases, the calculated phase diagram becomes in reasonable qualitative agreement to the experimental one in the $T-P$ region corresponding to ices Ih, II, III, V, and VI. The calculation of the phase diagram in the quantum and classical limits shows that the most important quantum effect is the stabilization of ice II due to its lower zero-point energy when compared to that one of ices Ih, III, and V. @ 2013 AIP Publishing LLC. [http://dx.doi.org/10.1063/1.4818875]
\end{abstract}

\section{INTRODUCTION}

Sixteen different crystalline ice phases have been identified so far in the phase diagram of water. ${ }^{1}$ In all phases, except ice $\mathrm{X}$, the water molecules are part of a network connected by $\mathrm{H}$-bonds. In most ice lattices there appears a unique H-bond network that fills the whole volume. However, four phases (ices VII, VIII, VI, and XV) are made of two identical and independent networks that interpenetrate one into another. Within a H-bond network, each oxygen atom is coordinated to four oxygen neighbors in a more or less distorted tetrahedral arrangement. The protons are distributed according to the Bernal-Fowler ice rules. They state that in a network there must be one and only one proton between two adjacent oxygen atoms and that each oxygen is part of two $\mathrm{OH}$ covalent bonds characteristic of the water molecule. ${ }^{2}$ These rules are compatible with either ordered or disordered spatial distributions of $\mathrm{H}$ atoms. In fact, order-disorder transitions have been observed for most pairs of ice phases (Ih-XI, III-IX, V-XIII, VI-XV, VII-VIII, XII-XIV). Only for H-ordered ice II and $\mathrm{H}$-disordered ices Ic and IV, the other member of the corresponding pair has not been yet experimentally found. ${ }^{3}$

A comprehensive review of the calculation of free energies of water phases with the thermodynamic integration (TI) method can be found in Ref. 4. The classical phase diagram of water, simulated with the rigid TIP4P/2005 model, shows a reasonable qualitative agreement to the experimental one, in

${ }^{\text {a) }}$ Electronic mail: ramirez@icmm.csic.es particular in the complex region of stability of ices Ih, II, III, $\mathrm{V}$, and VI. ${ }^{5}$ The coexistence of these ice phases has been also studied by quantum path integral simulations with the rigid TIP4PQ/2005 model. ${ }^{6}$ The phase diagram of ices Ih, II, and III was additionally investigated using a flexible water model (q-TIP4P/F) in the classical limit. ${ }^{7}$ Singer and Knight have analyzed the order-disorder transition in ices Ih-XI, III-IX, V-XIII, VI-XV, VII-VIII, and XII-XIV by the calculation of the small energy differences between the innumerable $\mathrm{H}$-bond configurations possible in a large simulation cell. ${ }^{8}$ Since the lattice energy is a scalar, it can be related to topological properties of the H-bond configurations that are invariant to the symmetry operations of the lattice. This link between H-bond topology and energetics is used to extrapolate from electronic calculations on small unit cells to larger cells that approximate the thermodynamic limit. Thus accurate results for the order-disorder transitions in ice are obtained just by focusing on the dependence of the lattice energy with the H-bond configurations. The vibrational energy was assumed to play a secondary role in these transitions. H-bond order-disorder transitions are understood as discontinuous changes in the H-bond topologies sampled by the system, while the oxygen lattice changes minimally. Note that for phase transitions other than order-disorder ones, the change in the oxygen lattice is drastic. Therefore, for such transitions the vibrational free energy is expected to play a significant role.

The quasi-harmonic $(\mathrm{QH})$ approximation $(\mathrm{QHA})$ allows us to compute the free energy of a solid as an analytic function of the volume and the temperature for a given interatomic 
potential. ${ }^{9}$ The prediction of the volume, enthalpy, kinetic energy, and heat capacity of ices Ih, II, and III by the QHA has been compared to results of quantum path integral molecular dynamics (PIMD) simulations using the q-TIP4P/F model. A remarkable overall agreement was found in temperature $(T)$ and pressure $(P)$ ranges up to $300 \mathrm{~K}$ and $1 \mathrm{GPa}$, respectively. ${ }^{10}$ Moreover, the QHA offers a simple alternative to TI methods to study the phase diagram of solid phases.

The phase boundary between ices VII and VIII has been studied by a QHA in a 16-molecule supercell with ab initio density-functional theory (DFT) calculations of total energies and phonon frequencies. ${ }^{11}$ The calculation shows that the coexistence line in the $P-T$ diagram has negative Clapeyron slope and a noticeable isotope effect, both facts in good agreement to experimental data. The phase diagram of ices Ih, II, and III has been recently calculated by a QHA. ${ }^{12}$ The studied models were based on both flexible (q-TIP4P/F) and rigid (TIP4P/2005 and TIP4PQ/2005) descriptions of the water molecule. The QHA was able to reproduce, for each of the studied models, the available coexistence lines Ih-II, II-III, and Ih-III of the phase diagrams derived by TI methods. Moreover, the simplicity of the QHA allowed to uncover new information by considering conditions that had not been treated in previous TI simulations. In particular, for the typical cell sizes used in phase coexistence simulations, the averaging over the proton disorder of ice III was an essential step to obtain a converged phase diagram. Thus, the common procedure of using only one randomly selected ice III structure makes the calculated phase diagram affected by an uncontrolled factor that can be highly significant for the final result. ${ }^{12}$

The purpose of the present paper is to derive the phase diagram of all experimentally known ice phases of ice (except ice $\mathrm{X}$ ) using the QHA in combination with the flexible q-TIP4P/F water model. ${ }^{13}$ The layout of the manuscript is as follows. A summary of the employed computational conditions is presented in Sec. II. The generation of the ice structures is introduced in Sec. III. The effect of H-disorder averaging in the lattice energy is discussed in Sec. IV. The calculated phase diagram of ice is compared to the experimental one in Sec. V. The pressure dependence of the free energy of several ice phases is presented in Sec. VI. A comparison of classical and quantum phase diagrams is given in Sec. VII. The paper closes with the conclusions.

\section{COMPUTATIONAL CONDITIONS}

The employed QHA has been introduced in Refs. 10 and 12 . We present here a brief summary. The Helmholtz free energy of an ice phase with $N$ water molecules in a cell of volume $V$ and at temperature $T$ is given by

$$
F(V, T)=U_{S}(V)+F_{v}(V, T)-T S_{H}+\Delta U_{\text {ave }},
$$

where $U_{S}(V)$ is the static zero-temperature classical energy, i.e., the minimum of the potential energy when the volume of the cell is $V . F_{v}(V, T)$ is the vibrational contribution to $F$. In the quantum limit it is given by

$$
F_{v}(V, T)=\sum_{k}\left(\frac{\hbar \omega_{k}}{2}+\frac{1}{\beta} \ln \left[1-\exp \left(-\beta \hbar \omega_{k}\right)\right]\right) .
$$

Here, $\beta$ is the inverse temperature: $1 / k_{B} T . \omega_{k}$ are the wavenumbers of the harmonic lattice vibrations for the volume $V$, with $k$ combining the phonon branch index and the wave vector within the Brillouin zone. The anharmonicity of the interatomic potential enters in the QHA only through the volume dependence of $\omega_{k}$. In the classical limit, the vibrational contribution amounts to

$$
F_{v, c l a}(V, T)=\sum_{k} \frac{1}{\beta} \ln \left(\beta \hbar \omega_{k}\right) .
$$

The entropy $S_{H}$ and the energy $\Delta U_{\text {ave }}$ are related to the disorder of hydrogen and they vanish for the ordered ice phases (i.e., ices XI, II, IX, XIII, XV, VIII, and XIV). $S_{H}$ was estimated by Pauling for fully disordered phases as ${ }^{14}$

$$
S_{H}=N k_{B} \ln \frac{3}{2} .
$$

A comparison of the Pauling estimate to accurate numerical determinations has been recently presented for several ice phases. ${ }^{15} \Delta U_{\text {ave }}$ is a constant energy that depends on the average of the lattice energy over the proton disorder of the ice phase (see below). The Gibbs free energy, $G(T, P)$, is obtained by seeking for the volume, $V_{\min }$, that minimizes the function $F(V, T)+P V$, as

$$
G(T, P)=F\left(V_{\min }, T\right)+P V_{\min } .
$$

The implementation of the QHA for an ice phase follows these steps: ${ }^{10,12}$

(i) Find the reference cell that minimizes the static energy $U_{S}$. This minimization implies optimization of both cell shape and atomic positions. The resulting volume is $V_{r e f}$ and the corresponding static energy is $U_{S, r e f}$.

(ii) Only for $\mathrm{H}$-disordered phases: generate a random set of structures with different $\mathrm{H}$-configurations and calculate the constant energy $\triangle U_{\text {ave }}$ as

$$
\triangle U_{\text {ave }}=\bar{U}_{S, r e f}-U_{S, r e f},
$$

where $\bar{U}_{S, r e f}$ is the average of the static lattice energy for the generated set of $\mathrm{H}$-isomers, while $U_{S, \text { ref }}$ is the lattice energy of the reference cell considered in step (i). The number of random $\mathrm{H}$-isomers is set so large that the estimated error of the mean value $\bar{U}_{S, r e f}$ is lower than $0.02 \mathrm{~kJ} / \mathrm{mol}$. It is also sensible to take as reference cell in step (i) the structure whose lattice energy $U_{S, r e f}$ is closest to the average $\bar{U}_{S, r e f}$.

(iii) Select a grid of 50 volumes in a range of interest $\left[V_{\min }, V_{\max }\right]$. The ice cell with volume $V_{i}$ is set by isotropic scaling of the reference cell. Subsequently, each ice cell is held fixed while minimizing the static energy $U_{S}\left(V_{i}\right)$ with respect to the atomic positions. The crystal phonons, $\omega_{k}\left(V_{i}\right)$, are obtained after the minimization.

(iv) Calculate the function $F\left(V_{i}, T\right)$ by Eq. (1). The minimum of $F\left(V_{i}, T\right)$ as a function of $V$ is determined by a fit to a 5 th degree polynomial in $V$.

The phase diagram of ice is derived by a brute force method, i.e., given a state point $(T, P)$ one calculates the Gibbs 
free energy of all ice phases. The stable phase is selected as the one with the lowest value of $G$.

The crystal phonon calculation has been performed by the small-displacement method. ${ }^{16,17}$ For the flexible water model the atomic displacement employed in this work is $\delta x$ $=10^{-6} \AA$ along each Cartesian direction. We have used a $\Gamma$ sampling $(\mathbf{k}=\mathbf{0})$ of the crystal phonons, which is a reasonable approximation for the sizes of the employed supercells. The Lennard-Jones interaction between oxygen centers was truncated at a distance of $r_{c}=8.5 \AA$, and standard long-range corrections for both potential energy and pressure were computed assuming that the pair-correlation function is unity for $r>r_{c}{ }^{18}$ Long-range electrostatic potential and forces were calculated with the Ewald method.

The assumption of isotropic scaling of the reference cell made in step (iii) was checked for ice II in Ref. 12. By relaxing this constraint the QHA free energy of ice II changes slightly, by about $0.01 \mathrm{~kJ} / \mathrm{mol}$, having a small effect in the phase diagram. $^{12}$

\section{ICE STRUCTURES}

Supercells of similar size to those employed in recent simulations $^{6,7}$ have been used in the $\mathrm{QH}$ derivation of the phase diagram. In Table I we summarize the crystallographic references used in the generation of the ice structures. Supercells are defined by translation vectors applied along the crystallographic axes of the lattice. The total number $N$ of water molecules generated in the supercell is also given. The potential energy $\left(U_{S, r e f}\right)$ and volume $\left(V_{\text {ref }}\right)$ obtained in the minimization of the supercell structures with the q-TIP4P/F model are presented, together with the volume interval $\left[V_{\min }, V_{\max }\right]$ used in the QHA for each phase. The optimized reference cells and the corresponding fractional coordinates of the water molecules for each studied ice phase are made available in the supplementary material. ${ }^{19}$

The algorithm proposed by Buch et al. ${ }^{20}$ was applied for the random generation of full proton disordered structures with vanishing cell dipole moment of ices Ih, Ic, III, IV, V, VI, VII, and XII. The reason for choosing a vanishing cell dipole moment is that the disordered ice phases are not ferroelectric. In the case of ice III and ice V the neutron diffraction experiments show the existence of partial H-disorder, i.e., fractional H-occupancies different from 0.5. ${ }^{21}$ The Buch's algorithm has been then slightly modified for the generation of random structures with partial $\mathrm{H}$-disorder. ${ }^{22}$ For these phases the proton disorder entropy is somewhat lower than the Pauling result, $S_{H}$. We have employed the estimations of $0.9 S_{H}$ and $0.94 S_{H}$ for ices III and V, respectively. ${ }^{22}$

In the generation of the crystal structures the following particularities were considered. For ice Ih the reference supercell was orthorhombic with parameters $(4 a, 3 \sqrt{3} a, 3 c)$, with $(a, c)$ being the standard hexagonal parameters of ice $\mathrm{Ih}^{23}$ For ice XI, the H-ordered form of ice Ih, we have generated two different structures with crystal symmetry $C m c 2_{1}$ and $P n a 2_{1}$. The former corresponds to the experimental phase ice XI. ${ }^{24}$ The latter is associated with the global energy minimum predicted by TIP4P-like models that is not in accord with experiment. ${ }^{20}$ For ice $\mathrm{V}$ the orthorhombic cell setting $(\mathbf{a}, \mathbf{b}, \mathbf{c})$ used in Ref. 21 corresponds to the space group symbol $A 2 / a$, but was changed here to a more standard setting $(\mathbf{c},-\mathbf{b}, \mathbf{a})$ with space group symbol $C 2 / c .^{25}$ In the case of the high pressure phases' ices VII and VIII, the energy minimization of a flexible supercell did not lead to a stable crystal lattice at zero pressure. To overcome this difficulty the form of the supercell was constrained to that one obtained by classical

TABLE I. The space group of the studied phases is shown with the reference used to generate the ice supercell. H-disordered phases (except ices IV and Ic) are found in a row immediately above the H-ordered counterpart. The number of water molecules in each supercell is $N$. The static lattice energy ( $\left.U_{S, r e f}\right)$ and volume $\left(V_{r e f}\right)$ of the optimized supercells were derived with the q-TIP4P/F model. [ $\left.V_{\min }, V_{\max }\right]$ is the volume interval studied by the QHA. The data for ices III and V correspond to both full and partial H-disorder. Energy unit is kJ/mol, volumes in $\AA^{3} / \mathrm{molecule}$.

\begin{tabular}{|c|c|c|c|c|c|c|c|}
\hline Space symmetry & Supercell & H-disorder & $N$ & $U_{S, r e f}$ & $V_{\text {ref }}$ & $V_{\min }$ & $V_{\max }$ \\
\hline Ic $(F d \overline{3} m)^{31}$ & $(3,3,3)$ & Yes & 216 & -62.00 & 31.00 & 20.44 & 35.02 \\
\hline Ih $\left(P 6_{3} / m m c\right)^{32}$ & $(4,3 \sqrt{3}, 3)$ & Yes & 288 & -61.98 & 30.96 & 29.47 & 35.05 \\
\hline $\mathrm{XI}\left(C m c 2_{1}\right)^{24}$ & $(4,3,3)$ & No & 288 & -61.95 & 31.03 & 29.48 & 35.05 \\
\hline XI $\left(P n a 2_{1}\right)^{33}$ & $(3,3,4)$ & No & 288 & -62.02 & 30.90 & 29.30 & 34.96 \\
\hline II $(R \overline{3})^{34}$ & $(3,3,3)$ & No & 324 & -60.84 & 24.14 & 21.75 & 27.31 \\
\hline III $\left(P 4_{1} 2_{1} 2\right)$ & $(3,3,3)$ & Yes (full) & 324 & -60.96 & 24.90 & 23.58 & 28.14 \\
\hline III $\left(P 4_{1} 2{ }_{1} 2\right)^{21}$ & $(3,3,3)$ & Yes (partial) & 324 & -60.72 & 25.05 & 24.07 & 28.31 \\
\hline IX $\left(P 4_{1} 2{ }_{1} 2\right)^{35}$ & $(3,3,3)$ & No & 324 & -61.52 & 24.63 & 23.55 & 27.83 \\
\hline $\mathrm{IV}(R \overline{3} c)^{36}$ & $(2,2,2)$ & Yes & 128 & -59.77 & 22.10 & 18.49 & 24.31 \\
\hline $\mathrm{V}(C 2 / c)$ & $(2,3,3)$ & Yes (full) & 504 & -60.28 & 22.84 & 20.55 & 25.81 \\
\hline $\mathrm{V}(C 2 / c)^{21}$ & $(2,3,3)$ & Yes (partial) & 504 & -60.04 & 22.99 & 20.80 & 25.98 \\
\hline XIII $\left(P 2{ }_{1} / a\right)^{37}$ & $(2,3,3)$ & No & 504 & -60.15 & 23.15 & 20.83 & 26.16 \\
\hline $\mathrm{VI}\left(P 4_{2} / n m c\right)^{38}$ & $(3,3,4)$ & Yes & 360 & -59.58 & 21.44 & 18.14 & 22.51 \\
\hline $\mathrm{XV}(P \overline{1})^{30}$ & $(3,3,4)$ & Yo & 360 & -59.43 & 21.53 & 18.43 & 22.61 \\
\hline VII $(P n \overline{3} m)^{38}$ & $(6,6,6)$ & Yes & 432 & -53.08 & 19.57 & 14.02 & 20.98 \\
\hline $\mathrm{VIII}\left(I 4_{1} / a m d\right)^{39}$ & $(5,5,3)$ & No & 600 & -53.19 & 19.47 & 13.92 & 20.83 \\
\hline $\mathrm{XII}(I \overline{4} 2 d)^{40}$ & $(2,2,4)$ & Yes & 288 & -60.06 & 21.99 & 18.81 & 24.19 \\
\hline $\operatorname{XIV}\left(P 2{ }_{1} 2{ }_{1} 2_{1}\right)^{37}$ & $(2,2,4)$ & No & 192 & -60.62 & 21.90 & 18.32 & 24.09 \\
\hline
\end{tabular}


NPT simulations of ices VII and VIII using a flexible cell at $P=2 \mathrm{GPa}$ and $T=50 \mathrm{~K}$, i.e., at conditions where instability problems are fully absent. Then subsequent energy minimizations of ices VII and VIII maintaining the rigid form of the ice cell do always lead to stable crystal structures even at volumes corresponding to small negative pressures.

The validity of the QHA in ice is restricted by the presence of anharmonic effects not included in the approximation. Such effects are expected to increase at high temperature. A direct check of the QHA is the comparison to numerical simulations that fully consider the anharmonicity of the interatomic interactions. PIMD results of the density of ice phases for a number of state points are compared to the corresponding QHA as well as to available experimental data in Table II. The studied state points appear in a temperature range between 77 and $300 \mathrm{~K}$. We find a reasonable agreement between PIMD and QHA densities even at high temperature. Similar temperature behavior was reported for the volume, enthalpy, and heat capacity of ices Ih, II, and III in Ref. 12 up to $300 \mathrm{~K}$. Note that the thermal energy at $300 \mathrm{~K}$ corresponds to a wavenumber $\left(k_{B} T / \hbar\right)$ of about $200 \mathrm{~cm}^{-1}$, so that at $300 \mathrm{~K}$ most ice phonons remain in their vibrational ground state. In particular, those related to the molecular stretching and bending modes, as well as the H-bond librations. ${ }^{10}$ The comparison between calculated and experimental ice densities in Table II displays a satisfactory overall agreement. The largest deviation is found for the high-pressure ice VII, where the calculated q-TIP4P/F density is about 5\% lower than the experimental one. Such error has been previously reported in ice VII by classical and quantum Monte Carlo (MC) simulations using TIP4P-like models. ${ }^{26,27}$

\section{DISORDER AVERAGING}

An interesting practical question is the importance of proton disorder in the stability of $\mathrm{H}$-disordered cells. Two op-

TABLE II. Ice densities derived by PIMD simulations of ice phases using the $\mathrm{q}-\mathrm{TIP} 4 \mathrm{P} / \mathrm{F}$ model are compared to the corresponding QHA, as well as to experimental data. Simulation results for ices III and V correspond to cells with full $\mathrm{H}$-disorder. Computational conditions for the PIMD simulations are the same as in Ref. 10. The employed H-isomer of ice III had a static lattice energy of $-60.86 \mathrm{~kJ} / \mathrm{mol} .{ }^{12}$ Density unit is $\mathrm{g} \mathrm{cm}^{-3}$.

\begin{tabular}{lcccccc}
\hline \hline Phase & $T(\mathrm{~K})$ & $P(\mathrm{GPa})$ & $\rho$ (PIMD) & $\rho$ (QHA) & $\rho$ (Exp.) & Ref. \\
\hline Ih & 250 & 0.0 & 0.925 & 0.917 & 0.920 & 41 \\
II & 123 & 0.0 & 1.190 & 1.191 & 1.190 & 42 \\
III (full) & 250 & 0.28 & 1.168 & 1.177 & 1.165 & 43 \\
IV & 110 & 0.0 & 1.290 & 1.296 & 1.272 & 36 \\
V (full) & 237.7 & 0.53 & 1.269 & 1.272 & 1.271 & 44 \\
VI & 225 & 1.1 & 1.397 & 1.382 & 1.373 & 38 \\
VII & 300 & 10 & 1.783 & 1.785 & 1.880 & 45 \\
VIII & 77 & 2.4 & 1.590 & 1.592 & 1.628 & 38 \\
IX & 165 & 0.28 & 1.187 & 1.191 & 1.194 & 43 \\
XI $\left(C m c 2_{1}\right)$ & 77 & 0.0 & 0.931 & 0.930 & 0.934 & 46 \\
XII & 260 & 0.5 & 1.301 & 1.299 & 1.292 & 40 \\
XIII & 80 & 0.0 & 1.241 & 1.242 & 1.244 & 37 \\
XIV & 250 & 0.28 & 1.308 & 1.311 & 1.332 & 37 \\
XV & 80 & 0.0 & 1.329 & 1.336 & 1.326 & 30 \\
\hline \hline
\end{tabular}

posite strategies have been used to address this point. The first one is to use a cell so small that an explicit calculation of the internal energy of all existing $\mathrm{H}$-isomers is possible. The lattice symmetry may be exploited by considering only symmetry inequivalent $\mathrm{H}$-isomers with the corresponding multiplicity. ${ }^{11}$ This approach leads to an exact average over proton disorder, but at the cost of introducing an unspecified finite size effect as a consequence of the small unit cell.

The second strategy is to define a large supercell and calculate the internal energy of a single $\mathrm{H}$-isomer of the disordered phase. The average over proton disorder is then introduced ad hoc by adding the proton disorder entropy $S_{H}$, as in Eq. (1). An implicit assumption here is that the supercell is so large that both the lattice energy and the vibrational free energy of the single $\mathrm{H}$-isomer have already converged with respect to any change in the proton configuration. Such a change is understood to be compatible with the Bernal-Fowler rules and with the corresponding (full or partial) proton disorder. This procedure has been adopted in most TI studies of the phase diagram of ice. ${ }^{5-7,28}$ In addition, we have already commented in the Introduction the approach by Singer and Knight, extrapolating the results of small unit cells to larger ones to study order-disorder transitions. ${ }^{8}$

Note that the assumption of the convergence of the lattice energy with the proton disorder in a large supercell is only correct in the thermodynamic limit, as the relative fluctuation of thermodynamic quantities is expected to decrease as $1 / \sqrt{N}$. For finite $N$, the Buch's algorithm will produce a set of $\mathrm{H}$-isomers with different lattice energies and therefore also different statistical weights. Only in the thermodynamic limit will the Buch's algorithm produce proton configurations with the same statistical weight, as it was assumed by Pauling in his estimation of the residual entropy of ice in Eq. (4). In this respect, Eq. (6) can be understood as a finite size correction for the estimation of the lattice energy associated with the thermodynamic limit. ${ }^{12}$

The simplicity of the QHA renders possible to check the convergence of the lattice energy, for a given cell size, with respect to the proton disorder. In our previous $\mathrm{QH}$ study of ices Ih and III, the convergence of the static lattice energy, $U_{S, \text { ref }}$, was studied for a small set of six random $\mathrm{H}$-isomers in a cell with 324 molecules. $^{12}$ In the following we present a more accurate account of the convergence of $U_{S, r e f}$ for ice III.

\section{A. Ice III}

In Fig. 1 we have represented the results of $U_{S, r e f}$ for a set of 50 random $\mathrm{H}$-isomers of ice III. The supercell contains 324 water molecules with full $\mathrm{H}$-disorder. The static lattice energy $U_{S, r e f}$ is plotted as a function of the corresponding cell volume, $V_{r e f}$. For comparison, we also show the data obtained when ice III has partial H-disorder, and the value for ice IX, the $\mathrm{H}$-ordered counterpart of ice III. The minimized lattice energy, $U_{S, r e f}$, and volume, $V_{\text {ref }}$, are related in a nearly linear way. We note that all isomers having partial $\mathrm{H}$-disorder display larger static energy than those with full $\mathrm{H}$-disorder. 


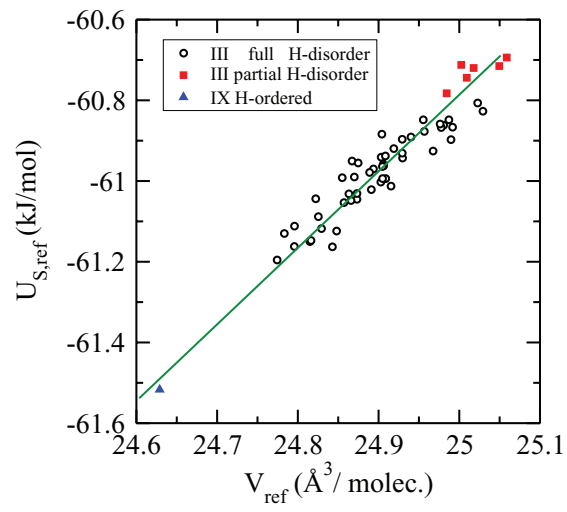

FIG. 1. Lattice energy and volume of a set of H-isomers of ice III generated randomly according to the Bernal-Fowler ice rules. The H-isomers display either full (open circles) or partial (closed squares) $\mathrm{H}$-disorder. The results were derived with the q-TIP4P/F model for a supercell with 324 molecules. The close triangle shows the result for ice IX, the H-ordered counterpart of ice III. The line is a guide to the eye.

An important result of Fig. 1 is that for the employed supercell with 324 molecules the dispersion of $U_{S, \text { ref }}$ is rather large $(\sim 0.4 \mathrm{~kJ} / \mathrm{mol})$. We have chosen a threshold of 0.05 $\mathrm{kJ} / \mathrm{mol}$ as criterion to qualify if a given energetic difference can be considered as significant in the calculation of the phase diagram. Then, following this criterion, the spreading of the lattice energy of ice III may appreciably affect the phase diagram whenever it is calculated with a single random $\mathrm{H}$-isomer of ice III. As a remedy to this uncertainty, the average term $\triangle U_{\text {ave }}$ was introduced in Eq. (1) to improve the convergence of the internal energy of ice with respect to the proton disorder. ${ }^{12}$

The effectiveness of this averaging procedure is illustrated in Fig. 2. It shows the phase diagram of ices Ih, II, and III calculated for six random $\mathrm{H}$-isomers of ice III with the qTIP4P/F model. The lattice energy $U_{S, \text { ref }}$ of these H-isomers
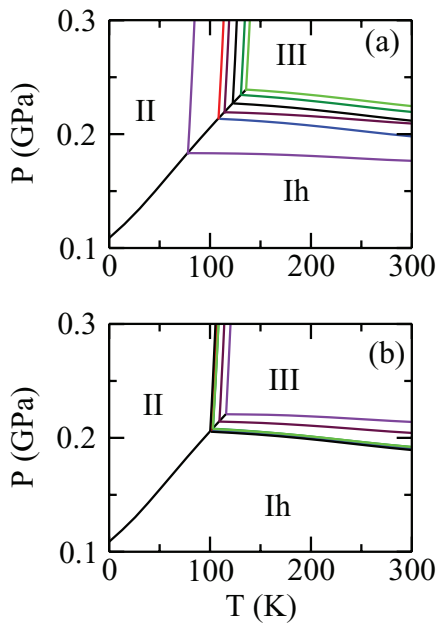

FIG. 2. (a) QHA phase diagram of ices Ih, II, and III. The coexistence lines were calculated for six random $\mathrm{H}$-isomers of ice III with full $\mathrm{H}$-disorder. The free energy of ice III does not include the disorder averaging term $\Delta U_{\text {ave }}$ of Eq. (1). (b) Same diagram after including the disorder averaging term $\Delta U_{\text {ave }}$ in the free energy of ice III. Note the decrease in the dispersion of the coexistence lines. All results are derived with the q-TIP4P/F model in the quantum limit. scatters in an interval of about $0.3 \mathrm{~kJ} / \mathrm{mol}$. In Fig. 2(a) the free energy of ice III was calculated without the H-disorder averaging term $\left(\triangle U_{\text {ave }}\right)$ of Eq. (1). These results are identical to those presented in Fig. 4 of Ref. 12. Coexistence lines of different ice III structures are clearly separated. The region of stability of ices Ih, II, and III appears strongly dependent on the $\mathrm{H}$-disorder. The phase diagram obtained after considering the term $\triangle U_{\text {ave }}$ in Eq. (1) is shown in Fig. 2(b). The spreading of the coexistence lines is now appreciably reduced. The remaining dispersion is related to the vibrational free energy that is also affected by the disorder of protons in the employed supercell. However, this effect of $\mathrm{H}$-disorder in the vibrational energy is comparatively less important than in the lattice energy. A similar conclusion has been presented in the analysis of order-disorder transitions in Ref. 8.

\section{B. Other disordered phases}

The mean static lattice energy, $\bar{U}_{S, r e f}$, and its standard deviation, $\sigma\left(U_{S, r e f}\right)$, was calculated by sampling a set of random H-isomers for all H-disordered phases (Ih, Ic, III, IV, V, VI, VII, and XII). The results are summarized in Table III. A large value of the standard deviation, $\sigma$, implies that $\mathrm{H}-$ disorder strongly affects the value of the static energy, $U_{S, r e f}$, of the supercell and therefore also the stability of the ice phase. The largest value of $\sigma$ corresponds to ice III with full $\mathrm{H}$-disorder $(\sigma=0.1 \mathrm{~kJ} / \mathrm{mol})$. Accordingly the static energy, $U_{S, \text { ref }}$, of a single random $\mathrm{H}$-isomer of ice III can be found in an energy window of about $4 \sigma \sim 0.4 \mathrm{~kJ} / \mathrm{mol}$, as shown in Fig. 1.

The standard deviations $\sigma\left(U_{S, r e f}\right)$ given in Table III decrease along the series of ices: III (full disorder) $>$ XII $>$ V (full disorder) $>$ III (partial disorder) $>$ IV $>$ VI. For these phases, the no consideration of H-disorder averaging may introduce arbitrary shifts in the lattice energy larger than 0.05 $\mathrm{kJ} / \mathrm{mol}$, at least for the supercell sizes employed here. For ices Ih, Ic, and VII the energetic effect of H-disorder is smaller than this threshold so that it can be safely neglected for the studied supercells.

TABLE III. Result of the averaging of the lattice energy, $U_{S, \text { ref }}$, of $\mathrm{H}$-disordered ices using the q-TIP4P/F model. For each phase we show the number of molecules in the supercell $(N)$, the number of random H-isomers in the average $\left(N_{\text {ave }}\right)$, the mean value of the lattice energy, $\bar{U}_{S, \text { ref }}$, and its standard deviation, $\sigma\left(U_{S, r e f}\right)$. Results for ices III and $\mathrm{V}$ are shown for both full and partial H-disorder. The last two columns are in units of $\mathrm{kJ} / \mathrm{mol}$.

\begin{tabular}{lcccc}
\hline \hline Ice & $N$ & $N_{\text {ave }}$ & $\bar{U}_{S, \text { ref }}$ & $\sigma\left(U_{S, r e f}\right)$ \\
\hline Ih & 288 & 6 & -61.98 & 0.00 \\
Ic & 216 & 9 & -62.00 & 0.00 \\
III (full) & 324 & 50 & -60.98 & 0.10 \\
III (partial) & 324 & 6 & -60.73 & 0.03 \\
IV & 128 & 9 & -59.77 & 0.02 \\
V (full) & 504 & 10 & -60.27 & 0.03 \\
V (partial) & 504 & 6 & -60.03 & 0.01 \\
VI & 360 & 10 & -59.57 & 0.02 \\
VII & 432 & 6 & -53.08 & 0.01 \\
XII & 288 & 9 & -60.07 & 0.04 \\
\hline \hline
\end{tabular}



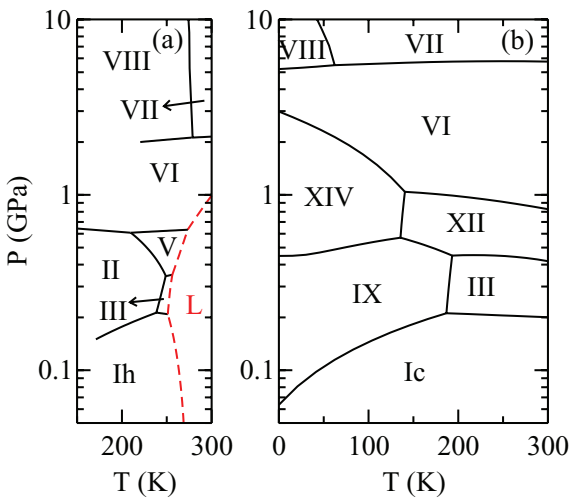

FIG. 3. Phase diagram of ice. (a) Experimental result from Ref. 1. The broken line is the boundary between ice and liquid (L) water. Full lines denote coexistence conditions between ice phases. (b) QHA result derived in the quantum limit with the q-TIP4P/F potential model.

For ices III and V, the mean lattice energy, $\bar{U}_{S, r e f}$, is significantly larger $(\sim 0.24 \mathrm{~kJ} / \mathrm{mol})$ in the case of partial than in the case of full $\mathrm{H}$-disorder. This behavior is in contradiction to the experimental finding that ices III and V display both partial H-disorder. ${ }^{21}$ This unphysical result is in line with the reported limitations of the effective potentials to reproduce the energetics of the H-bond rearrangement in the ice phases. ${ }^{8,20}$

Our analysis on the disorder averaging of the lattice energy of ice has omitted several factors that might be relevant. The consideration of reference cells with non-zero dipole moment should increase the standard deviation, $\sigma\left(U_{S, r e f}\right)$, and also affect the mean static lattice energy of the cell. This behavior has been demonstrated in the classical MC simulation of the dielectric constant of ice using several water models. ${ }^{29}$ Another factor is the fractional occupation of $\mathrm{H}$-sites in the ice structures with partial $\mathrm{H}$-disorder (ices III and V) that may depend on the employed water model. This was shown in Ref. 29 where the fractional occupancies $\left(\mathrm{H}_{\alpha}, \mathrm{H}_{\beta}\right)$ of ice III, experimentally found as $(0.35,0.5),{ }^{21}$ change to $(0.5,0.25)$ by using the TIP4P/2005 model. ${ }^{29}$ In our treatment of partial disorder in ices III and V we have considered only fractional occupancies derived from the experimental data.

\section{QUASI-HARMONIC PHASE DIAGRAM}

The experimental phase diagram of ice at temperatures in the range $[150,300] \mathrm{K}$ and pressures below $10 \mathrm{GPa}$ is presented in Fig. 3(a). ${ }^{1}$ The broken line shows the boundary between ice an liquid water. Full lines are the coexistence lines measured for the ice phases. In the displayed region there appear seven different stable ice phases: Ih, II, III, V, VI, VII, and VIII. Note that ice XV, the H-ordered counterpart of ice VI, appears at temperatures lower than $130 \mathrm{~K}$ and is not shown in the figure. ${ }^{30}$

The $\mathrm{QH}$ result derived with the $\mathrm{q}-\mathrm{TIP} 4 \mathrm{P} / \mathrm{F}$ model in the quantum limit is presented in Fig. 3(b). The free energy was calculated for the ice phases listed in Table I by using Eqs. (1) and (5). There are striking differences between the calculated phase diagram and the experimental data. The main deviations of the model are as follows:
- the most stable phase at low pressures is ice Ic instead of ices Ih or XI,

- the H-ordered ice IX is stable in the region where experimentally appears the H-ordered ice II,

- the H-ordered ice XIV occupies part of the stability region of the $\mathrm{H}$-disordered ice VI and $\mathrm{H}$-ordered ice $\mathrm{XV}$,

- the H-disordered ice XII occupies the stability region of ice $\mathrm{V}$.

We stress that ices II and V are not stable phases in the calculated phase diagram. Instead the ice polymorphs IX, XIV, and XII occupy large regions of stability. Such behavior has not been reported in any TI simulations of the phase diagram of ice using models based on the TIP4P potential. ${ }^{4-7}$

One may wonder if these unexpected findings are a pathology of the QHA. Against this point of view it can be argued that the QH phase diagram of ices Ih, II, and III studied in Ref. 12 is in reasonable agreement with TI simulations. Deviations found between $\mathrm{QH}$ and TI methods for several models (rigid TIP4P/2005 and TIP4PQ/2005, as well as flexible q-TIP4P/F) were more likely due to structural differences in the supercell employed for ice III than to limitations of the QHA. ${ }^{12}$ For this reason, we consider plausible that the QHA is providing valid information about the potential model for $(T, P)$ regions and ice phases that have not been previously studied by TI methods. Thus, the understanding of these unexpected findings is worth the effort.

In addition to the information displayed in the phase diagram of Fig. 3(b), it is interesting to know the free energy differences between stable and metastable phases in several regions of the phase diagram. Free energy differences lower than the threshold of $0.05 \mathrm{~kJ} / \mathrm{mol}$ are considered within the numerical error of the method and therefore will not be given a large physical significance. In Sec. VI we present a closer look into the calculated free energies at state points where the most stable phase is either ice Ic, IX, XIV, or XII.

\section{QUASI-HARMONIC FREE ENERGIES}

\section{A. $T=0 \mathrm{~K}$ and $P=0$}

The QH Gibbs free energy, $G_{0}$, of an ice phase at $T=0$ $\mathrm{K}$ and $P=0$ is the sum of two energy contributions:

$$
G_{0}=U_{S, 0}+U_{Z, 0} .
$$

$U_{S, 0}$ is the static lattice energy for the equilibrium volume, $V_{0}$, that includes the averaging term for the proton disorder,

$$
U_{S, 0}=U_{S}\left(V_{0}\right)+\triangle U_{\text {ave }} .
$$

$U_{Z, 0}$ is the zero-point energy calculated as

$$
U_{Z, 0}=\sum_{k} \frac{\hbar \omega_{k}\left(V_{0}\right)}{2} .
$$

In Table IV we collect the values of $U_{S, 0}, U_{Z, 0}$, and $V_{0}$ of the ice phases studied with the q-TIP4P/F model.

The most stable (i.e., lowest $G_{0}$ ) phases are ices Ic, XI, and Ih. Although the predicted order of increasing stability is $\mathrm{Ic}>\mathrm{XI}\left(\right.$ Pna $\left._{1}\right)>\mathrm{XI}\left(C m c 2_{1}\right)>\mathrm{Ih}$, the free energy 
differences between them are lower than the threshold of $0.05 \mathrm{~kJ} / \mathrm{mol}$. Such small differences are also conserved at higher pressures and temperatures. Therefore, the stability of ices Ih, Ic, and XI is nearly identical for the employed model. For the rest of the paper, we refer to ice Ih as representative for these ice phases with almost equal free energy.

Then, when compared with other ice polymorphs, ice Ih displays several distinct properties at $T=0 \mathrm{~K}$ and $P=0$. It has the largest volume $\left(V_{0}\right)$ per water molecule, the lowest lattice energy $\left(U_{S, 0}\right)$, and the highest zero-point energy $\left(U_{Z, 0}\right)$ of all ice phases. The leading factor for the stability of ice Ih at $T=0 \mathrm{~K}$ and $P=0$ is its lowest lattice energy.

It is interesting to note that the nuclear quantum effect causes an expansion of the equilibrium volume of ice at low temperatures. This anharmonic effect in the structure of ice is predicted by the QHA due to zero-point contribution to the free energy in Eq. (7). This energy term is absent in the classical limit, where the free energy is equal to the static lattice energy at $T=0 \mathrm{~K}$. Therefore, the equilibrium volume, associated with the minimum of the Gibbs free energy, is different in the quantum and classical limits. We find that the quantum volume, $V_{0}$, of the ice phases at $T=0 \mathrm{~K}$ (see Table IV) is typically $4 \%$ larger than those ones derived in the classical limit (see $V_{\text {ref }}$ values in Table I).

\section{B. Increasing pressure at $T=0 \mathrm{~K}$}

The values of $G_{0}$ and $V_{0}$ in Table IV allow us to rationalize the changes in the stability of the ice phases upon an increase of the pressure at $T=0 \mathrm{~K}$. A positive pressure will add a $P V$ term to the free energy $G_{0}$. Obviously, the larger the ice volume the larger the increase in the free energy. Then ice Ih (with the largest volume) will be destabilized with respect to all other ice phases upon an increase of pressure. Ice IX is the best candidate to become stable. It has the lowest value

TABLE IV. QH Gibbs free energy $\left(G_{0}\right)$ calculated at $T=0 \mathrm{~K}$ and $P=0$ with the q-TIP4P/F model in the quantum limit. The partition of $G_{0}$ into lattice $\left(U_{S, 0}\right)$ and zero-point $\left(U_{Z, 0}\right)$ energy is given. $V_{0}$ is the equilibrium volume in $\AA^{3} /$ molecule. The data for ices III and V correspond to full $\mathrm{H}$-disorder. Energy units are in $\mathrm{kJ} / \mathrm{mol}$.

\begin{tabular}{lcccc}
\hline \hline q-TIP4P/F & $G_{0}$ & $U_{S, 0}$ & $U_{Z, 0}$ & $V_{0}$ \\
\hline Ic & 6.97 & -61.77 & 68.74 & 32.35 \\
XI(Pna2 $\left.{ }_{1}\right)$ & 6.99 & -61.77 & 68.76 & 32.19 \\
XI(Cmc2 $\left.2_{1}\right)$ & 7.01 & -61.73 & 68.73 & 32.26 \\
Ih & 7.01 & -61.74 & 68.75 & 32.23 \\
IX & 7.22 & -61.40 & 68.62 & 25.60 \\
II & 7.47 & -60.60 & 68.08 & 25.11 \\
III (full) & 7.82 & -60.89 & 68.71 & 25.90 \\
XIV & 7.90 & -60.39 & 68.29 & 22.78 \\
XIII & 8.18 & -59.97 & 68.15 & 24.01 \\
V (full) & 8.28 & -60.07 & 68.35 & 23.72 \\
XII & 8.35 & -59.84 & 68.20 & 22.85 \\
IV & 8.60 & -59.56 & 68.17 & 22.93 \\
VI & 8.71 & -59.35 & 68.06 & 22.25 \\
XV & 8.74 & -59.21 & 67.95 & 22.34 \\
VIII & 14.05 & -52.81 & 66.86 & 20.55 \\
VII & 14.15 & -52.80 & 66.95 & 20.43 \\
\hline \hline
\end{tabular}

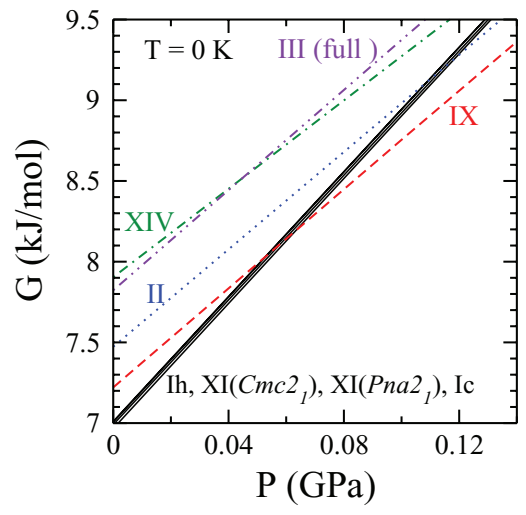

FIG. 4. Gibbs free energy, $G$, of the ice phases with lowest $G$ at $T=0 \mathrm{~K}$ and pressures below $0.14 \mathrm{GPa}$. The results correspond to the QHA using the q-TIP4P/F model. The curves for ices Ih, XI, and Ic are nearly identical at the displayed energy scale. A phase transition from ice Ih to ice IX is predicted at $P \sim 0.06 \mathrm{GPa}$.

of $G_{0}$ after that one of ice Ih, and its equilibrium volume is significantly lower $(20 \%)$ than that of ice Ih.

In Fig. 4 we have represented the pressure dependence up to $0.14 \mathrm{GPa}$ of the Gibbs free energy of the ice phases with lowest $G$ at $T=0 \mathrm{~K}$. We have plotted the free energies of ices Ic, Ih, and XI to show that their small free energy differences at $P=0$ are conserved as pressures increase. We observe that at low pressures the phase having the minimum free energy is ice Ih. However, as the pressure increases above $0.06 \mathrm{GPa}$, ice IX becomes more stable than ice Ih.

A further increase of the pressure will stabilize another ice phase with even lower volume than ice IX. In Fig. 5 we show the Gibbs free energy, $G$, of several ice phases in the range $0.42<P<0.48 \mathrm{GPa}$. The crossing of the free energy lines of ices IX and XIV at $0.45 \mathrm{GPa}$ is the fingerprint for a phase transition from ice IX to ice XIV.

Given a pressure $P$, for thermodynamic consistency in the low-temperature limit $(T \rightarrow 0 \mathrm{~K})$ one expects the stable ice polymorph to be an $\mathrm{H}$-ordered phase. This does not appear to be always the case in our calculations. Thus, at $P<60 \mathrm{MPa}$ we find cubic ice Ic to be the low $T$ stable phase. However, as indicated above, free-energy differences between

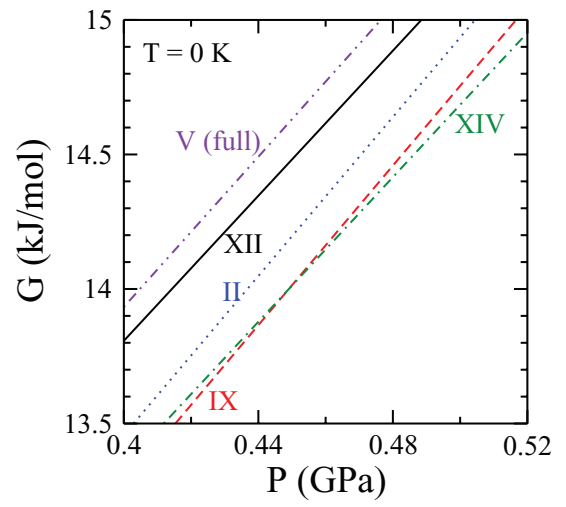

FIG. 5. Gibbs free energy, $G$, of the ice phase with lowest $G$ at $T=0 \mathrm{~K}$ and pressures in the interval $[0.4,0.52] \mathrm{GPa}$. The results are derived by the QHA using the q-TIP4P/F model. A phase transition from ice IX to ice XIV is predicted at $P \sim 0.45 \mathrm{GPa}$. 
ice Ic and the H-ordered ice XI are smaller than our sensitivity limit $(\sim 0.05 \mathrm{~kJ} / \mathrm{mol})$. Also, ice VI appears to be the stable phase at low temperatures in the region between 3 and $5 \mathrm{GPa}$. In this case one would expect the corresponding H-ordered phase (ice XV) to be the stable polymorph, but its free energy for $T \rightarrow 0 \mathrm{~K}$ is higher than that of ice VI. This problem may be related to the possibility that the $\mathrm{q}-\mathrm{TIP} 4 \mathrm{P} / \mathrm{F}$ potential could favor an $\mathrm{H}$-ordered pattern different from that corresponding to ice $\mathrm{XV}$, as those discussed earlier in the literature. ${ }^{30}$

\section{Stability of ice IX versus ice II}

A consequence of the stability of ice IX is that ice II does not appear (i.e., it is metastable) in the $\mathrm{QH}$ phase diagram of Fig. 3(b). This metastability of ice II disagrees obviously with the experimental phase diagram. In Figs. 4 and 5 the free energy $G$ of ice II is larger than that of ice IX. The difference is nearly independent of the pressure, as the $G(P)$ curves of ices IX and II are approximately parallel. If we measure the free energy difference between these phases at $T=0$ by the ordinates in the origin of Fig. 4 (i.e., the $G_{0}$ values of ices IX and II in Table IV), one gets

$$
\Delta G_{0}(\mathrm{II}-\mathrm{IX})=0.25 \mathrm{~kJ} / \mathrm{mol} \text {. }
$$

The positive value implies that ice IX is more stable than ice II. The energy partitioning of $G_{0}$ in Table IV shows that the leading term for the larger stability of ice IX is its lower lattice energy.

Note that if the $G(P)$ curve of ice IX was omitted from Fig. 4, then the first transition as a function of pressure would correspond to the crossing of the Ih and II free energy curves at $0.11 \mathrm{GPa}$. This Ih-II transition was shown in the study of the coexistence of ices Ih, II, and III in Fig. 2.

Given the large stability of ice IX predicted by the q-TIP4P/F model, we want to address the following question: Is this stability a consequence of the flexibility of the model or it has its origin in the TIP4P-character of the potential?

To this aim we have calculated the $\mathrm{QH}$ free energies of several ice phases by using the rigid TIP4P/2005 model. This model was parameterized for water simulations in the classical limit. In this limit, at $T=0 \mathrm{~K}$ and $P=0$, the Gibbs free energy and equilibrium volume of an ice phase are simply given by

$$
\begin{gathered}
G_{0, c l a} \equiv \bar{U}_{S, r e f}, \\
V_{0, c l a} \equiv V_{r e f} .
\end{gathered}
$$

For reference, the values of $G_{0, c l a}$ and $V_{0, c l a}$ calculated with the TIP4P/2005 model for ices Ih, XI, II, and XIV are shown in Table V. Note that in this case

$$
\Delta G_{0, c l a}(\mathrm{II}-\mathrm{IX})=0.58 \mathrm{~kJ} / \mathrm{mol} \text {. }
$$

This free energy difference between ices IX and II is even larger than that found for the flexible q-TIP4P/F model.

The classical QH phase diagram of ices Ih, II, and IX was calculated with the rigid TIP4P/2005 model at temperatures up to $300 \mathrm{~K}$ and pressures below $0.6 \mathrm{GPa}$. We find that ice IX is more stable than ice II in the whole studied $(T, P)$ region.
TABLE V. Gibbs free energy $\left(G_{0, c l a}\right.$ in $\left.\mathrm{kJ} / \mathrm{mol}\right)$ calculated with the rigid TIP4P/2005 model for several ice phases at $T=0 \mathrm{~K}$ and $P=0$ in the classical limit. $V_{0, c l a}$ is the equilibrium volume in $\AA^{3} /$ molecule.

\begin{tabular}{lcc}
\hline \hline TIP4P/2005 & $G_{0, \text { cla }}$ & $V_{0, \text { cla }}$ \\
\hline Ih & -62.99 & 31.34 \\
IX & -62.71 & 24.87 \\
II & -62.13 & 24.30 \\
XIV & -61.72 & 22.10 \\
\hline \hline
\end{tabular}

Therefore, ice II is metastable in the classical phase diagram of the TIP4P/2005 model. This result is identical to that found for the flexible q-TIP4P/F model. Our conclusion is that the large stability of ice IX is a property of the TIP4P-character of the model, and not a consequence of the added molecular flexibility.

We believe that limitations inherent to the exploration of the phase diagram by TI methods are the reason why the stability of ice IX and the metastability of ice II have not been detected in previous studies using TIP4P-like models. ${ }^{4,6}$ An advantage of the QHA is that the brute force calculation of free energies allows a thorough exploration of state points for all ice phases.

\section{Increasing pressure at $T=250 \mathrm{~K}$}

An unexpected result of the calculated phase diagram at temperatures around $250 \mathrm{~K}$ is that ice XII, the H-disordered counterpart of ice XIV, occupies the stability region where one would expect to find ice V as stable phase (see Fig. 3). The pressure dependence of the free energy of the ice phases with lowest $G$ is presented in Fig. 6 at $250 \mathrm{~K}$. The crossing of the $G(P)$ curves of ices III and XII at $P=0.45 \mathrm{GPa}$ indicates that ice XII becomes the stable phase at $250 \mathrm{~K}$ for pressures higher than $0.45 \mathrm{GPa}$.

It is interesting to note that at the pressures shown in Fig. 6 the free energy of ice $\mathrm{V}$ (with full $\mathrm{H}$-disorder) is only slightly higher $(\sim 0.06 \mathrm{~kJ} / \mathrm{mol})$ than that of ice XII. Besides, ice XII displays lower volume than ice V. Therefore, an increase in the pressure will always stabilize ice XII with

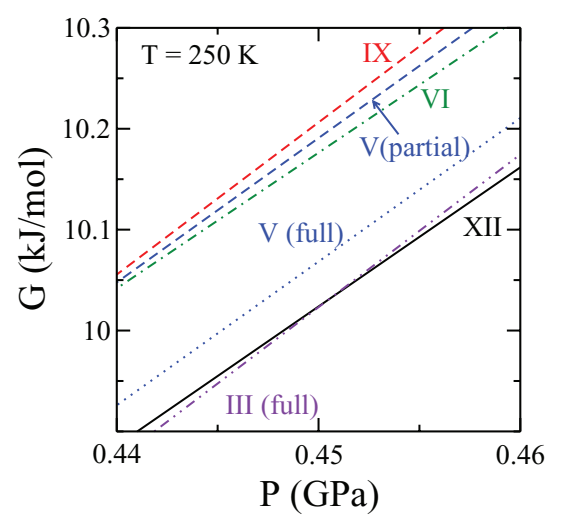

FIG. 6. Gibbs free energy, $G$, of the ice phase with lowest $G$ at $T=250 \mathrm{~K}$ and pressures in the interval [0.44,0.46] GPa. The results correspond to the q-TIP4P/F model and the QHA. A phase transition from ice III to ice XII appears at $P \sim 0.45 \mathrm{GPa}$. 
respect to ice V. At $T=250 \mathrm{~K}$ the $\mathrm{QHA}$ predicts that the coexistence pressure for ices $\mathrm{V}$-XII is $0.33 \mathrm{GPa}$. The equilibrium volumes at this state point $(T=250 \mathrm{~K}, P=0.33 \mathrm{GPa})$ are $23.1 \AA^{3} /$ molecule and $23.8 \AA^{3} /$ molecule, for ices XII and $\mathrm{V}$, respectively.

Note that at $250 \mathrm{~K}$ the pressure interval where ice $\mathrm{V}$ appears as stable phase in the experimental phase diagram is about $[0.35,0.6] \mathrm{GPa}$ (see Fig. 3(a)). In this pressure range the q-TIP4P/F model predicts that the free energy difference between ices $\mathrm{V}$ and XII increases from a vanishingly small value (at $0.35 \mathrm{GPa}$ ) to a maximum value of $0.1 \mathrm{~kJ} / \mathrm{mol}$ (at $0.6 \mathrm{GPa}$ ). Therefore, free energy differences between ices XII and V are relatively small. Similar values for the free energy of ices V and XII have been already reported for the TIP4P/2005 model at $P=0.5 \mathrm{GPa}$ by TI simulations in the classical limit. ${ }^{4}$

\section{QUANTUM VS. CLASSICAL PHASE DIAGRAM}

The QH phase diagram of ice shown in Fig. 3(b) for the q-TIP4P/F model is characterized by the stability of ices IX, XII, and XIV over large regions of the $(T, P)$ plane. However, these phases have not been reported as stable ones in previous studies. For this reason, our calculated phase diagram has little resemblance to previous ones derived by using rigid TIP4P-like potentials in combination with TI methods. 4, 6, 28

Then, it is interesting to recalculate our phase diagram of ice under omission of the phases IX, XII, and XIV. In addition, we will consider ice Ih as unique representative of the phases Ih, Ic, and XI.

The quantum limit of the new phase diagram of the qTIP4P/F model is presented in Fig. 7. Now we find the following stable phases: Ih, II, III, V, VI, VII, and VIII. Both ices III and V correspond to full $\mathrm{H}$-disordered lattices. This phase diagram is in reasonable qualitative agreement to the experimental one. Moreover, it is also in reasonable agreement to those phase diagrams derived for TIP4P-like potentials. ${ }^{4,6,28}$

Concerning the thermodynamic consistency of these results for $T \rightarrow 0 \mathrm{~K}$, we emphasize that the H-disordered ices Ih and VI cannot strictly be the low-temperature stable phases for any pressure $P$. For this question, the arguments are the same as those given above in Sec. VI B and are not repeated here.

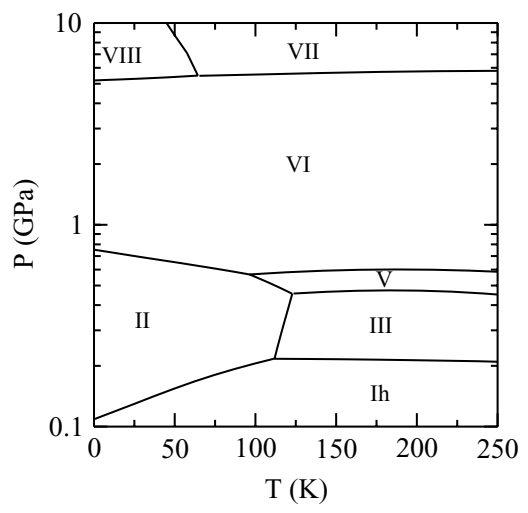

FIG. 7. QHA of the phase diagram of ice using the q-TIP4P/F model in the quantum limit. Ices III and $\mathrm{V}$ are full $\mathrm{H}$-disordered. The following ice phases were omitted from the QH calculation: ices IX, XIV, and XII.

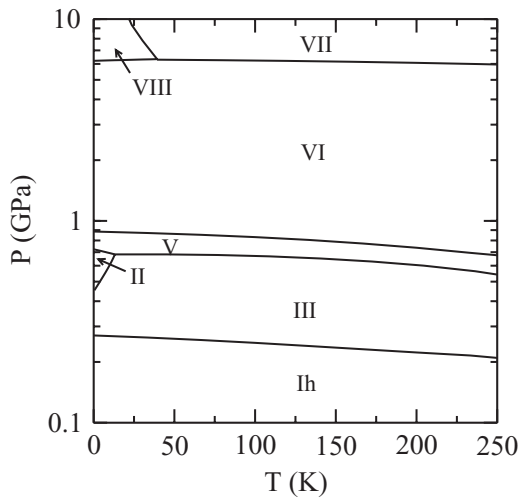

FIG. 8. The phase diagram of Fig. 7 is calculated with the q-TIP4P/F model in the classical limit.

Our results in Fig. 7 include the averaging of the lattice energy over H-disorder for ices III, V, and VI. None of the previously published phase diagrams calculated with TIP4Plike potentials include any kind of disorder averaging. In fact, different single $\mathrm{H}$-isomers of ice III seem to have been employed for the calculations with several TIP4P-like potentials (i.e., TIP4P, ${ }^{28}$ TIP4P $/ 2005,{ }^{4}$ and TIP4PQ/2005 ${ }^{6}$ ). If the ice III structure employed for each potential model has a different H-configuration, then the lattice energy of ice III may be affected by an uncontrolled factor. This situation makes it difficult to draw definitive conclusions about differences found in calculated phase diagrams with different $\mathrm{H}$-isomers of ice III. This uncertainty should affect not only ice III, but also the stability region of other phases (Ih, II, V) having a boundary with ice III.

It is interesting to compare the quantum phase diagram in Fig. 7 with that one calculated within the classical limit (Fig. 8). The main difference between both limits is related to the stability region of ice II. It is much larger in the quantum case. The origin of this quantum effect is related to the lower zero-point energy of ice II, in comparison to ices Ih, III, and $\mathrm{V}$ (see the $U_{Z, 0}$ values given in Table IV). The quantum stabilization of ice II has been already discussed in Ref. 12.

An additional difference between quantum and classical phase diagrams is that the coexistence lines that are nearly horizontal (i.e., Ih-III, III-V, V-VI) appear shifted to higher pressures in the classical limit. This effect has been also reported in TI simulations with the rigid TIP4PQ/2005 model. ${ }^{6}$ The rising of the pressure occurs at all studied temperatures. This quantum effect is most easily explained at $T=0 \mathrm{~K}$. Let us consider ices VI and V as an example. In the quantum limit, the free energy difference between ices VI and V at $T=0 \mathrm{~K}$ and $P=0$ is (see the $G_{0}$ data in Table IV)

$$
\triangle G_{0}(\mathrm{VI}-\mathrm{V})=0.4 \mathrm{~kJ} / \mathrm{mol} \text {. }
$$

However, in the classical limit one gets using Eq. (11) and the values of Table I,

$$
\triangle G_{0, c l a}(\mathrm{VI}-\mathrm{V})=0.7 \mathrm{~kJ} / \mathrm{mol} .
$$

In both cases $\Delta G_{0}>0$, i.e., ice VI is less stable than ice V. However, in the quantum case $\Delta G_{0}$ is lower. The reason is that the zero-point energy (see the $U_{Z, 0}$ data in Table IV) tends to stabilize ice VI with respect to ice $\mathrm{V}$ by $\sim-0.3 \mathrm{~kJ} / \mathrm{mol}$. 
On the other side, although the volume of ice VI is lower than that of ice $\mathrm{V}$ (see $V_{0}$ data in Table IV), the volume differences are found to be nearly the same in the quantum $\left(\Delta V_{0}=-1.5 \AA^{3} /\right.$ molecule $)$ and classical cases $\left(\Delta V_{0, c l a}\right.$ $=-1.4 \AA^{3} /$ molecule, see the $V_{\text {ref }}$ data in Table I).

By increasing the pressure, the phase with lower volume (ice VI) will become more stable. The pressure needed to stabilize ice VI with respect to ice $\mathrm{V}$ is roughly given by

$$
P \sim-\frac{\Delta G_{0}}{\Delta V_{0}}
$$

Note that while the denominator is similar in both quantum and classical limits, the numerator is lower in the quantum case. Therefore, the coexistence pressure for ices V-VI at $T$ $=0 \mathrm{~K}$ is reduced in the quantum limit with respect to the classical one.

A similar argument explains why the transitions Ih-III and III-V are displaced to higher pressures in the classical case. $^{6}$

\section{CONCLUSIONS}

The phase diagram of ice has been studied by a quasiharmonic approximation using the flexible q-TIP4P/F model of water. The simplicity of this approach allows us to include all known ice polymorphs (except ice $\mathrm{X}$ ) and all state points for $T<400 \mathrm{~K}$ and $P<10 \mathrm{GPa}$.

Surprisingly the simple QHA seems to be accurate enough to reproduce free energy differences between ice phases, in spite of the large complexity and variety in their crystal structures. This conclusion about the success of the QHA is derived using a simple model potential, but its validity is expected to be largely independent of the model. Therefore, it opens a route for the study of the whole phase diagram of ice by ab initio electronic structure methods.

The H-disorder of many ice phases is an additional complication in the calculation of their phase diagram. The QHA has allowed us to quantify the influence of this effect. The disorder averaging of the lattice energy of ice III has been proven to be important to obtain a converged phase diagram, at least using TIP4P-like models and ice III supercells with full $\mathrm{H}$-disorder. Disorder averaging of vibrational free energies has been shown to be comparatively less important. In addition to ice III, the disorder averaging of the lattice energy of ices XII and V has been shown to be also significant for the ice stability.

We stress that phase diagrams calculated using a single random $\mathrm{H}$-isomer of ice III may be affected by an uncontrolled energetic factor that can be highly significant in the final result. An immediate consequence of this is that comparison of phase diagrams calculated using a single, but different, $\mathrm{H}$-isomer of ice III might not be physically sound. The reason is that the stability of the disordered phase may depend strongly on the employed $\mathrm{H}$-isomer.

The $\mathrm{QH}$ phase diagram of ice with the flexible q-TIP4P/F model has been calculated by performing a disorder averaging of the lattice energy of the $\mathrm{H}$-disordered ice phases. We have found an unexpected large stability of several ice phases, especially the H-ordered ices IX and XIV, and also the H-disordered ice XII. The presence of these phases in the calculated phase diagram implies that both ices II and $\mathrm{V}$ are metastable phases with the q-TIP4P/F model. This finding disagrees with the experimental phase diagram. We have checked that ice IX remains more stable than ice II if the phase diagram is calculated using the rigid TIP4P/2005 model in the classical limit. Our conclusion is that the larger stability of ice IX with respect to ice II is a property related to the TIP4Pcharacter of the model and not to the explicit treatment of the molecular flexibility.

The QH free energy and volume of several ice phases have been analyzed at $T=0 \mathrm{~K}$ and $P=0$. The free energy has been partitioned into lattice and zero-point energies. These contributions are important magnitudes in the analysis of the stability of the ice phases as a function of pressure.

By excluding ices IX, XIV, and XII from the calculation, we find that the phase diagram of the q-TIP4P/F model shows qualitative agreement to both experimental and previously simulated ones by using TIP4P-like models. The comparison of the quantum and classical limits shows several differences. The most important ones are the increase in the stability of ice II and the shift of the coexistence lines III-V and V-VI to lower pressures in the quantum case. Similar conclusions were reached previously in Ref. 6. Differences in the zero-point energies of the ice phases provide an explanation for these effects.

\section{ACKNOWLEDGMENTS}

This work was supported by MEC (Spain) through Grant No. FIS2012-31713, and by Comunidad Autónoma de Madrid through Project No. MODELICO-CM/S2009ESP1691.

${ }^{1}$ A. Dunaeva, D. Antsyshkin, and O. Kuskov, Solar System Research 44, 202 (2010).

${ }^{2}$ J. D. Bernal and R. H. Fowler, J. Chem. Phys. 1, 515 (1933).

${ }^{3}$ C. G. Salzmann, P. G. Radaelli, B. Slater, and J. L. Finney, Phys. Chem. Chem. Phys. 13, 18468 (2011).

${ }^{4}$ C. Vega, E. Sanz, J. L. F. Abascal, and E. G. Noya, J. Phys.: Condens. Matter 20, 153101 (2008).

${ }^{5}$ J. L. F. Abascal and C. Vega, J. Chem. Phys. 123, 234505 (2005).

${ }^{6}$ C. McBride, E. G. Noya, J. L. Aragones, M. M. Conde, and C. Vega, Phys. Chem. Chem. Phys. 14, 10140 (2012).

${ }^{7}$ S. Habershon and D. E. Manolopoulos, Phys. Chem. Chem. Phys. 13, 19714 (2011).

${ }^{8}$ S. J. Singer and C. Knight, Adv. Chem. Phys. 147, 1 (2011).

${ }^{9}$ G. P. Srivastava, The Physics of Phonons (Adam Hilger, Bristol, 1990).

${ }^{10}$ R. Ramírez, N. Neuerburg, M.-V. Fernández-Serra, and C. P. Herrero, J. Chem. Phys. 137, 044502 (2012).

${ }^{11}$ K. Umemoto, R. M. Wentzcovitch, S. de Gironcoli, and S. Baroni, Chem. Phys. Lett. 499, 236 (2010).

${ }^{12}$ R. Ramírez, N. Neuerburg, and C. P. Herrero, J. Chem. Phys. 137, 134503 (2012).

${ }^{13}$ S. Habershon, T. E. Markland, and D. E. Manolopoulos, J. Chem. Phys. 131, 024501 (2009).

${ }^{14}$ L. Pauling, J. Am. Chem. Soc. 57, 2680 (1935).

${ }^{15}$ C. P. Herrero and R. Ramírez, Chem. Phys. Lett. 568-569, 70 (2013).

${ }^{16}$ G. Kresse, J. Furthmüller, and J. Hafner, Europhys. Lett. 32, 729 (1995).

${ }^{17}$ D. Alfè, G. D. Price, and M. J. Gillan, Phys. Rev. B 64, 045123 (2001).

${ }^{18}$ J. K. Johnson, J. A. Zollweg, and K. E. Gubbins, Mol. Phys. 78, 591 (1993).

${ }^{19}$ See supplementary material at http://dx.doi.org/10.1063/1.4818875 for a set of ascii files with the supercells of the studied ice phases. For each file, the first line contains the number of atoms, volume, and potential energy of the supercell calculated with the q-TIP4P/F model. The second line 
includes the supercell parameters $a, b, c$ (in $\AA$ ) and $\alpha, \beta, \gamma$ (in degrees). The following lines contain the atomic symbol and fractional coordinates of all atoms.

${ }^{20}$ V. Buch, P. Sandler, and J. Sadlej, J. Phys. Chem. B 102, 8641 (1998).

${ }^{21}$ C. Lobban, J. L. Finney, and W. F. Kuhs, J. Chem. Phys. 112, 7169 (2000).

${ }^{22}$ L. G. MacDowell, E. Sanz, C. Vega, and J. L. F. Abascal, J. Chem. Phys. 121, 10145 (2004).

${ }^{23}$ J. A. Hayward and J. R. Reimers, J. Chem. Phys. 106, 1518 (1997).

${ }^{24}$ A. J. Leadbetter, R. C. Ward, J. W. Clark, P. A. Tucker, T. Matsuo, and H. Suga, J. Chem. Phys. 82, 424 (1985).

${ }^{25} \mathrm{~T}$. Hahn, International Tables for Crystallography, Vol. A: Space Group Symmetry (Kluwer Academic Publishers, Dordrecht, 1983).

${ }^{26}$ J. L. Aragones, M. M. Conde, E. G. Noya, and C. Vega, Phys. Chem. Chem. Phys. 11, 543 (2009).

${ }^{27}$ C. McBride, C. Vega, E. G. Noya, R. Ramírez, and L. M. Sesé, J. Chem. Phys. 131, 024506 (2009).

${ }^{28}$ E. Sanz, C. Vega, J. L. F. Abascal, and L. G. MacDowell, Phys. Rev. Lett. 92, 255701 (2004).

${ }^{29}$ J. L. Aragones, L. G. MacDowell, and C. Vega, J. Phys. Chem. A 115, 5745 (2011).

${ }^{30}$ C. G. Salzmann, P. G. Radaelli, E. Mayer, and J. L. Finney, Phys. Rev. Lett. 103, 105701 (2009).

${ }^{31}$ H. König, Z. Kristallogr. 105, 279 (1944).

${ }^{32}$ S. W. Peterson and H. A. Levy, Acta Crystallogr. 10, 70 (1957).
${ }^{33}$ T. K. Hirsch and L. Ojamäe, J. Phys. Chem. B 108, 15856 (2004).

${ }^{34}$ B. Kamb, W. C. Hamilton, S. J. LaPlaca, and A. Prakash, J. Chem. Phys. 55, 1934 (1971)

${ }^{35}$ S. J. LaPlaca, W. C. Hamilton, B. Kamb, and A. Prakash, J. Chem. Phys. 58, 567 (1973)

${ }^{36}$ H. Engelhardt and B. Kamb, J. Chem. Phys. 75, 5887 (1981).

${ }^{37}$ C. G. Salzmann, P. G. Radaelli, A. Hallbrucker, E. Mayer, and J. L. Finney, Science 311, 1758 (2006).

${ }^{38}$ W. F. Kuhs, J. L. Finney, C. Vettier, and D. V. Bliss, J. Chem. Phys. 81, 3612 (1984).

${ }^{39}$ J. M. Besson, P. Pruzan, S. Klotz, G. Hamel, B. Silvi, R. J. Nelmes, J. S. Loveday, R. M. Wilson, and S. Hull, Phys. Rev. B 49, 12540 (1994).

${ }^{40}$ C. Lobban, J. L. Finney, and W. F. Kuhs, Nature (London) 391, 268 (1998).

${ }^{41}$ K. Röttger, A. Endriss, J. Ihringer, S. Doyle, and W. F. Kuhs, Acta Cryst. B 50, 644 (1994).

${ }^{42}$ A. D. Fortes, I. G. Wood, M. Alfredsson, L. Vočadlo, and K. S. Knight, J. Appl. Crystallogr. 38, 612 (2005).

${ }^{43}$ J. D. Londono, W. F. Kuhs, and J. L. Finney, J. Chem. Phys. 98, 4878 (1993).

${ }^{44}$ R. E. Gagnon, H. Kiefte, M. J. Clouter, and E. Whalley, J. Chem. Phys. 92, 1909 (1990).

${ }^{45}$ R. J. Hemley, A. P. Jephcoat, H. K. Mao, C. S. Zha, L. W. Finger, and D. E. Cox, Nature (London) 330, 737 (1987).

${ }^{46}$ C. M. B. Line and R. W. Whitworth, J. Chem. Phys. 104, 10008 (1996). 